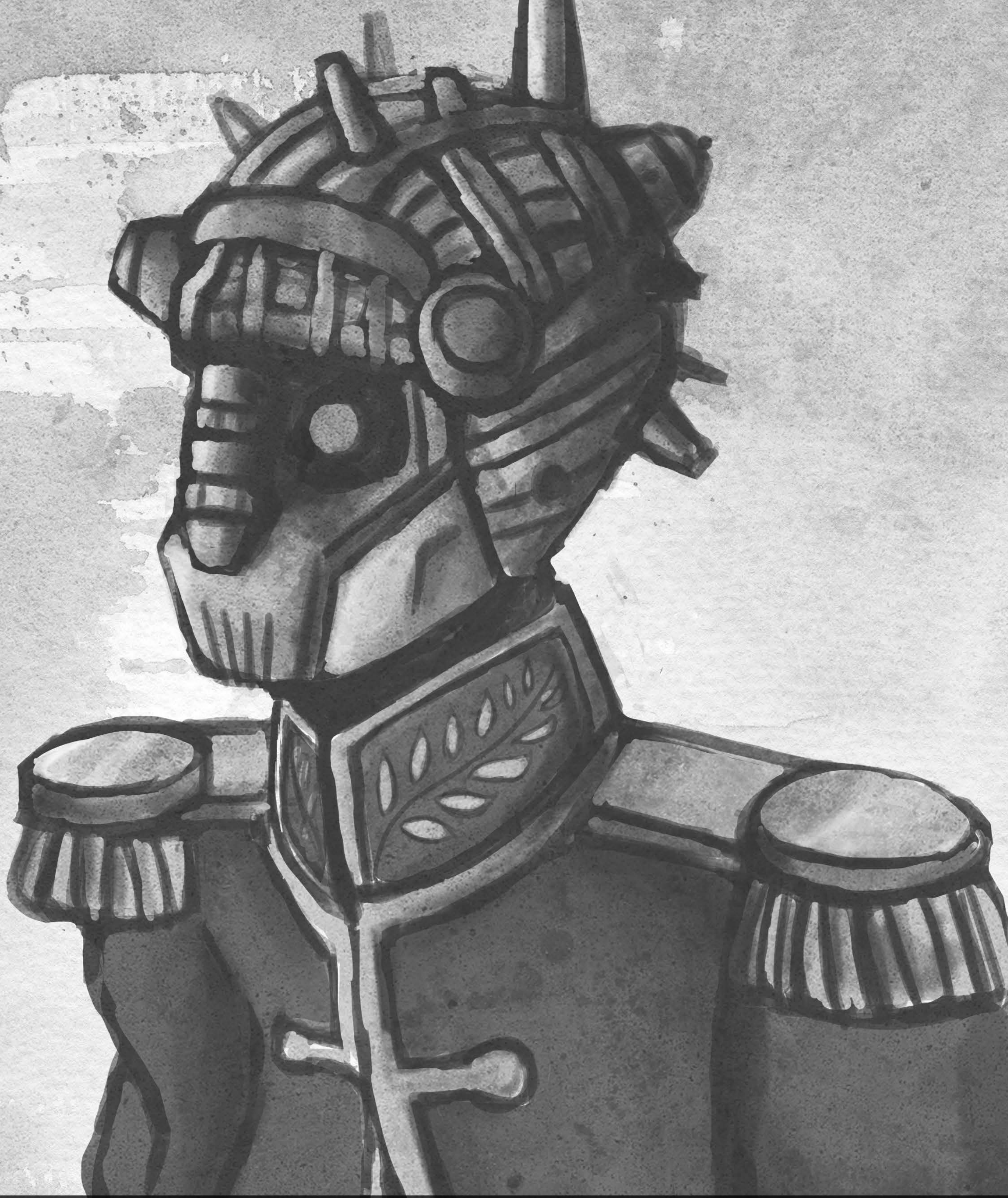

Entre la formación de la opinión pública y la movilización: el proceso independentista de Cartagena de Indias 


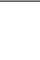




\section{Entre la formación de la opinión pública y la movilización: el proceso independentista de Cartagena de Indias}

Fecha de recepción: 13 de julio de 2011

Fecha de aceptación: 3 de septiembre de 2011

\section{José Alejandro Cepeda Jiménez}

Universidad Sergio Arboleda

joscep@yahoo.com

Magíster en Análisis de problemas políticos,

económicos e internacionales contemporáneos.

\section{Camilo Andrés Montoya Pardo}

Universidad Sergio Arboleda

cmontoyapardo@gmail.com

Profesional en Política y relaciones internacionales.

\section{Resumen}

Este artículo pretende explicar la relación entre el proceso de conformación de la opinión pública y elementos de movilización social en Cartagena de Indias como variables fundamentales para su proceso independentista, la cual fue posible bajo una nueva estructura política que reemplazó al régimen Monárquico, consecuencia directa de la revolución del 11 de noviembre de 1811, cuando Cartagena declarara su independencia absoluta de España.

\section{Palabras clave}

Opinión pública, movilización social, Independencia, esfera pública, representación, acción colectiva.

\section{Magda Catalina Jiménez Jiménez}

Universidad Sergio Arboleda magda.jimenez@usa.edu.co

Maestra en Estudios latinoamericanos de la Universidad de Salamanca.

\section{Abstract}

This article seeks to explain the relationship between the process of the public opinion structure and elements of social mobilization in Cartagena de Indias as fundamental variables for its independence process - which was possible under a new political structure that replaced the Monarchic regime. All this was a direct consequence of the revolution of November 11th, 1811, when Cartagena declared absolute independence from Spain.

\section{Keywords}

Public Opinion, Social Movement, Independence, Public Sphere, Representation, Collective Action. 


\section{Introducción}

El objetivo de este artículo, es analizar cómo los indicios para la formación de la opinión pública en Cartagena, fueron manifestaciones de dos segmentos de la sociedad ligados por un sentimiento de inconformidad frente a una estructura social jerarquizada. La cooperación entre criollos blancos y el bajo clero, con los indios de Malambo y los negros, llevaron a la conformación de una acción colectiva que, a través de una movilización, abrió las puertas al proceso de conformación de una opinión pública.

Si bien en ciudades como Santafé la conformación de una opinión pública obedeció a la iniciativa de una subesfera intelectual que lo organizó en ámbitos privados llamados tertulias, en Cartagena de Indias la posibilidad de formación de un público ilustrado y el acceso a la educación era una situación compleja, debido a la ausencia de centros universitarios, siendo el destino común de la mayoría de los criollos blancos de la ciudad educarse en Santafé en colegios mayores como el del Rosario o el de San Bartolomé. Cartagena de Indias, representa un caso particular en los procesos de Independencia del Virreinato de la Nueva Granada, ya que si bien fue la primera ciudad en declararse independiente de España, sirvió de ejemplo para que otras provincias y ciudades como Santafé replicaran esta iniciativa. La explicación obedece a la tensión que protagonizarian aquellos simpatizantes de la Independencia definitiva de España y los simpatizantes de una autonomía limitada que renunciaba a autodeterminarse, respetando la soberanía, no como un concepto que naciera del pueblo, sino como lealtad al Rey Fernando VII, por causa de las abdicaciones de 1809 , profundizando la crisis de la Monarquía española.

Es en este contexto donde se encuentra un principio de conformación ${ }^{1}$ de la opinión

$1 \quad$ Habermas diferencia tres estratos escalonados en la consolidación de la opinión pública: a) La que se establece a un nivel cultural prelingüístico en el intercambio de gustos y aficiones dentro de los grupos informales, como amigos y compañeros de la misma generación, conocidos del barrio, afinidades dentro de la familia; b) la que circula en forma de declaraciones institucionales autorizadas, avaladas por un nombre y un prestigio social o político; c) la que es conducida por los ciudadanos que intervienen en un proceso de comunicación argumentativa, vehiculado internamente por medios participativos y externamente a través de los mass-media. pública, término que implica el reconocimiento previo de un derecho fundamental que sustenta su existencia: la libertad de expresión. Derecho que solamente se reconoce en el momento en que la esfera pública y la privada ya no se encuentran sobre un mismo plano donde era imposible identificarlas por separado. Asistimos entonces a un punto de inflexión en el reino español, donde se está estructurando "un nuevo orden político basado en el poder limitado y dividido, la garantía de derechos y libertades del individuo y la publicidad de la acción política" (Muñoz, 1992, p. 23). En Cartagena, puede identificarse a partir del 11 de noviembre de 1811, donde el acta de Independencia firmada ese día abre nuevos caminos hacia la libertad política y abona el camino hacia un concepto de igualdad.

Así, el nuevo orden político que se estaba erigiendo, pretendía debilitar la figura del Estado encarnada en un gobierno monárquico cuyos líderes eran representantes de los reyes. El historiador Francois Xavier Guerra, interpreta que "La vida política del antiguo régimen se desarrolla en dos lugares, la Corte y la ciudad, y según modalidades muy concretas -parcialidades y bando- competencia entre los cuerpos y entre las redes de patronazgo" (Guerra y Lempiere, 1998, p. 12).

Bajo estas circunstancias se incubó la revolución independentista en Cartagena de Indias. Sin embargo, era todavía lejana la consolidación de una idea de ciudadano moderno que pudiera expresar libremente sus opiniones o ideas, ya que aún subsistian interrogantes acerca de quién realmente podía ejercer o manifestar la opinión públicamente y quién realmente tenía el derecho de hacerlo. No sería sino hasta la declaración de igualdad de derechos del ciudadano que el mundo conocería el concepto de ciudadano moderno que gozaría libremente de derechos civiles y políticos.

Aunque lo anterior responde a una consideración positiva o ideal que debe caracterizar a una sociedad politica moderna, las condiciones políticas entre 1780 y 1811 en la ciudad de Cartagena, eran sustancialmente particulares, empezando porque la publicidad de la acción política no se daría sino hasta el año de 1808 -1809 , cuando la Junta Central española decidiera, en un acto sin precedentes, como sostiene Hocquellet (1998), "Fomentar el desarrollo de los medios públicos de difusión de ideas, como la prensa, que permita la constitución de una 
esfera pública, más autónoma en lo político capaz de juzgar sus actos y consecuentemente de criticarlos también" (p. 141). La decisión de paulatinamente hacer pública la acción política por parte de la Junta Central española, abrió el espacio a la identificación de un público informado de las decisiones públicas y políticas, pero no libremente facultado para incidir directamente en las decisiones del Gobierno. Lo que sí es innegable, es que fue una respuesta a la crisis de la corona española tras la abdicación de la familia real a favor de Napoleón I, que generó el rechazo del Reino y la reivindicación de su lealtad a Fernando VII.

\section{Hacia la formación de una publicidad de la información}

En ese sentido, los funcionarios de cabildos, comerciantes, y militares de la ciudad, empiezan a ser protagonistas en el proceso de formación de opiniones acerca de la conducta y decisiones administrativas del Gobierno a través de la publicación de noticias en gacetas y periódicos; ejemplo de ello es una publicación del sábado 5 de septiembre de 1812 en el periódico $E 1$ Efimero de Cartagena, en el que "Se exhorta a la ciudadanía para que abandere la defensa de la Constitución Política", en un ejercicio que buscaba dentro del pueblo una manifestación de legitimación del poder político.

Por lo tanto, los papeles públicos fijaron percepciones que ayudaron a establecer indudablemente una opinión, no en vano la proclama pública del coronel Pedro Labatut ${ }^{2}$ donde se dirige al pueblo cartagenero para defender la Patria y atacar al pueblo de Santa Marta que se oponía a la independencia definitiva de los españoles, creó entre los habitantes de la ciudad un elemento de identidad hacia la causa independentista y despertó los sentimientos más hostiles contra Santa Marta, el más sólido bastión de los promonárquicos.

Sin embargo, la propiedad del manejo de la información y su adecuación a los intereses políticos de la Junta Central española, es un elemento que debe evaluarse antes de llegar a la conclusión de que fue únicamente la élite

2 El 4 de noviembre de 1812, el Coronel Pedro Labatut se dirige al pueblo cartagenero y a sus soldados para exhortarlos a la defensa de la Patria y para liderar una acción militar especial que tenía como objetivo atacar al pueblo de Santa Marta que se oponía a la independencia definitiva de España. politica, en un proceso de formación descendente de la opinión pública, la fuente de formación de ideas, interpretaciones y posiciones por parte del pueblo de Cartagena. Si bien la información estaba en manos de una élite política que intervino en la creación de la Junta de Gobierno de Cartagena en mayo 22 de 1810, era evidente que ésta era una representación infima en comparación con la representación que tenian los peninsulares en cabeza del alto clero y la nobleza. Este problema de representación fue el detonante de una movilización que encontró su cimiento más fuerte en acciones colectivas realizadas no solamente por el vulgo -término para referirse el pueblo iletrado-, sino también por un segmento del público ilustrado como los criollos y el clero bajo, que se convertirian en los lideres del movimiento independentista.

Así, la estructura de oportunidad politica estaba dada para adelantar una movilización que garantizara la manifestación popular de un pueblo que no toleró vivir un segundo más en las mismas condiciones de desigualdad y de limitación de derechos impuestas por la Junta Central española. Tarrow sostiene que "La acción colectiva, prolifera cuando la gente adquiere acceso a los recursos necesarios para escapar a su pasividad habitual y encuentra la oportunidad de usarlo" (Tarrow, 2003, p. 109). No sólo la creación de verdaderos espacios públicos, sino el uso paulatino de ellos como escenarios de discución, serían las condiciones necesarias para pensar en la aparición de la opinión pública en Cartagena; en ese sentido, la Independencia representó una ruptura en la historia entre el Antiguo Régimen y una modernidad política dispuesta a la representación de los otros estamentos: negros mulatos $\mathrm{y}$ artesanos.

El proceso de consolidación del proyecto ilustrado en Cartagena empieza a manifestarse con la impresión y publicación de papeles públicos como El Argos Americano el 17 de septiembre de 1810, o la Gaceta de Cartagena el 16 de abril de 1812. Sin embargo, ese proceso de difusión de información formaba una opinión "sesgada" ya que como sostiene Hocquellet (1998): "La definición de ese público podía variar de acuerdo con el mensaje que quisiera comunicarle" (p. 140). A pesar de ello, empieza a reconocerse en Cartagena a la élite política, como un formador de opiniones respecto al manejo de los asuntos público-administrativos, a través de la publicidad e impresión de 
las noticias de Gobierno que gozaba hasta esta época de un carácter exclusivo.

Ahora bien, no puede considerarse a la élite política de la ciudad como la única protagonista de la conformación de una opinión pública; en una sociedad estructuralmente jerarquizada como la cartagenera, donde la exclusión era característica hacia los negros y los esclavos y donde las posibilidades de ascenso social eran transversales al aspecto racial, la presencia de una tensión social estructurara acciones y movilizaciones que reclamaran justicia de diversa indole, siendo las demandas sobre la representación más visibles y constantes a partir de estos años.

\subsection{El papel de la imprenta}

La imprenta como herramienta de difusión de la información debilitó la posibilidad del imperio español de monopolizar las fuentes para transmitir un mensaje adaptado a la conveniencia del régimen politico. Por ello, fue fundamental para esta investigación, el uso que se hizo en la ciudad de la imprenta, la cual pasó de estar bajo el control de los funcionarios reales a aquel que pudiera adquirirla. Ejemplo de esto fue la imprenta de Diego Espinosa, la cual puso de manifiesto a los habitantes de la ciudad la impresión de las obras de Antonio Nariño, incluyendo los Derechos del hombre y el ciudadano. Este tipo de publicidad tuvo incidencia en los espacios públicos políticos, tal como sucedió en 1799 cuando "las autoridades de Cartagena se alarman y se asombran al ver apiñadas en una esquina de la ciudad a una multitud que lee: ¡Infelices habitadores de Cartagena! ya es tiempo que rompamos el yugo que tanto nos oprime, acábese para esto el infame Gobierno que tanto nos abate" (Torres, 1946, p. 332).

Por la información publicada en este panfleto y pegada en una pared de la ciudad, encontramos la existencia de elementos críticos a las decisiones y politicas que ha tomado el Gobierno virreinal; por ello no sorprende que durante la época en que se presentó la abdicación de Fernando VII, la Junta Suprema Central y Gubernativa del Reino haya decidido avanzar hacia un proceso de legitimación de su poder frente al del invasor francés, permitiendo publicidad abierta a las acciones del Gobierno regente, en un ejemplo de contra-información reciproca.
Sin un control estricto de la circulación e información que publicaban las imprentas, incluyendo la de las instituciones reales, aumentó la impresión de gacetas y periódicos como El Hércules (el primero en el Virreinato de la Nueva Granada en solicitar la abolición de la pena de muerte), La Gaceta Oficial, El Independiente Progresista, entre muchos otros. Este fue un fenómeno opinativo que ningún criollo o peninsular previó. Por ello fue preferible lograr cierto nivel de institucionalización de la imprenta en la ciudad por parte del Gobierno regente con el fin de oficializar el carácter público de las acciones de la élite política; atrás quedaron decisiones como la del Virrey Antonio José Amar y Borbón frente al uso que debía dársele a la imprenta proveniente de Filadelfia quien Consiguió Real Orden para que no se usase de ella: fue sepultada y condenada a perderse, hasta que en 1808 fue puesta en uso para reimprimir los mentirosos papelotes que traia Sinllorente, a fin de deslumbrar a las gentes sobre el verdadero y fatal estado de la PenÍnsula (Torres, 1946, p. 333).

La decisión eminentemente política de hacer público el desempeño del Gobierno y el nuevo protagonismo de la élite política de la ciudad como formadores de opinión pública, encaja perfectamente en el segundo estrato de la consolidación de la opinión pública planteado por Habermas, ya que empieza a formarse una opinión "que circula en forma de declaraciones institucionales autorizadas, avaladas por un nombre y un prestigio social o político" (Ferrer, 2002 , p. 25). Sin embargo, la participación en todo este proceso por parte de diversos colectivos que habitaron Cartagena, permite establecer que la ciudad se encontró entre 1811 a 1812 , en un tránsito entre el segundo y tercer estrato en este proceso de conformación de la opinión en el que "La opinión pública es conducida por ciudadanos que intervienen en un proceso de comunicación argumentativa, vehiculado internamente por medios participativos" (Ferrer, 2002, p. 25).

Dentro de esta lógica, los cartageneros empezaron a fijar una percepción de la acción gubernamental; para Hocquellet (1998) este proceso evidencia que "la voluntad de provocar el interés general, responde en primer lugar al deseo de encontrar una legitimidad surgida de ese mismo interés general y crea un público para los asuntos políticos; es decir un destinatario colectivo para las manifestaciones del poder" (p. 141). Ese nuevo público buscaría la autodeterminación política con la declaración 
de la Provincia de Cartagena como Estado libre e independiente. Se trata de un primer momento de ruptura con la estructura politica del Antiguo Régimen y la apertura hacia una opinión pública capaz de incidir en lo público.

En ese orden de ideas, el proceso de conformación de la opinión pública en la ciudad no encontraría las condiciones necesarias para su estructuración, sino hasta el momento en que pudo identificarse una clara contraposición entre lo público y lo privado; es decir, en el momento en que la provincia asistió a un debilitamiento o modificación de las viejas estructuras de poder que caracterizaban a la Monarquía española. Esa contraposición entre lo público y lo privado nos lleva a retomar los conceptos de esfera pública y esfera privada planteados por Jurgen Habermas, que si bien no se adaptan de forma completa a los contextos iberoamericanos de fines del siglo XVIII, sí se evidencia una conformación y estructuración de ciertos elementos que consideramos fundamentales para este proceso.

Este proceso de conformación alcanzó su momento más álgido en los acontecimientos del 11 de noviembre de 1811 , cuando la creación del Acta y conformación de la Junta de Gobierno permitió un espacio de deliberación donde empezaron a criticar, argumentar y discutir sobre las estructuras administrativas. Es en este contexto, cuando el pueblo de Cartagena reconoció el desconocimiento de sus derechos, reafirmando su intención de reclamar que sus opiniones influyeran en la estructura del Gobierno monárquico.

El reconocimiento de este hecho como el fundamental no busca desconocer las manifestaciones o expresiones anteriores a éste, que podrian interpretarse como "opiniones públicas"; lo que se quiere reconocer es que una opinión o manifestación bajo una estructura despótica era interpretada como una opinión irrelevante y subvalorada, cuya capacidad de redefinir el destino de lo politico era escasa. Surge entonces un gran interrogante: ¿Qué otros elementos aparte de la revolución, ayudaron a la consolidación del concepto de opinión pública en la ciudad de Cartagena? La posible respuesta puede encontrarse en dos hechos: la implementación en la ciudad de la imprenta, y la decisión en el año 1808 y 1809 de cambiar el carácter privado de la acción gubernamental, porque las decisiones politicas y asuntos públicos empiezan a ser conocidos por el pueblo y se incluye en un proceso acelerado de conocimiento de información por la consolidación de la imprenta.

En este sentido, estamos de acuerdo en que "una condición necesaria para el desarrollo de la opinión pública es el papel de la prensa" (Muñoz, 1992, p. 35), así como el de las tertulias y las sociedades de lecturas o instituciones de la publicidad como las llama Habermas. En el caso de Cartagena de Indias, la presencia de este tipo de instituciones no fue predominante debido a que aquellos que tomaron el camino de la ilustración, optaron por la educación que ofrecian las universidades con sede en la capital del Virreinato ${ }^{3}$. Ahora bien, el rasgo comercial hizo de la ciudad un centro de intercambio intenso y ágil de información y comunicaciones, siendo los papeles públicos y gacetas que provenian de Europa y algunas ciudades del Caribe, los que empezaron a fijar opiniones públicas en la ciudad, proceso que se consolidó con la llegada de la imprenta.

Finalmente, en la ciudad existió un proceso de conformación de la opinión pública en la que no sólo intervino la élite política a través de la publicidad de las acciones del Gobierno, sino otros segmentos sociales que se convirtieron en actores politicos durante este proceso, como los artesanos y mulatos por parte de los sectores populares; todos estos colectivos a partir de acciones colectivas fueron estructurando una narrativa histórica para una movilización.

\section{Elementos de movilización social}

La segmentación social que se presentó en la ciudad no sólo entre la misma élite sino entre los negros, donde podian identificarse entre libres, esclavos o mulatos, fue consecuencia del proceso de mestizaje, tal como lo afirma Adelaida Sourdis, quien sostiene que: "Dada dicha estratificación, equivocarse sobre el grado de blancura de alguien, era una ofensa que podia generar fuerte enemistad" (De la Vega, 1998, p. 19).

3 A pesar de las incomodidades, la élite local en buena parte se educaba en otros lugares. El caso de acercarse a Santafé implicaba serias dificultades, pues el transporte era difícil y las relaciones con las autoridades centrales no eran fluidas. 
En esas circunstancias, el movimiento que propició la Independencia se convirtió para estos estamentos en un medio que podría garantizar la libertad y oportunidades de movilidad social. Y es que a pesar de la rigidez en las reglas de ascenso social, inclusive dentro de la misma élite, los lideres del movimiento independentista fueron personajes ilustrados como comerciantes y criollos blancos descendientes de españoles, relegados del libre acceso a los estamentos politicos más altos como el clero y la esfera militar debido a las politicas administrativas de las reformas borbónicas.

De allí que la afirmación del profesor Guerra respecto a que: "Es durante la época de la Revolución y de la independencia cuando aparece el espacio público moderno, sin que haya sido precedido -sobre todo en Américapor la constitución total de una esfera pública literaria" (Guerra y Lempiere, 1998, p. 14) puede aplicarse a este caso. Coincidimos entonces en que fue en esta coyuntura de la crisis de la Monarquía hispánica cuando maduraron las condiciones estructurales para que fuera posible un debate político acerca de la titularidad de la soberanía y con ello la aparición de una opinión pública.

El sentimiento de vacío de poder se erigió como una amenaza para la estabilidad y cohesión del imperio español; en ese sentido la formación de las juntas de Gobierno en el Virreinato fue una respuesta a esta crisis de soberanía en donde se debilitó la idea pactista de la Monarquía, el argumento ideal para que los cartageneros empezaran a estructurar una oportunidad política para hacer posible una autodeterminación. En ese sentido, compartimos el argumento sobre la transformación que operó en esta ciudad, ya que de ser: Un movimiento que sólo pretendió en un principio aplicar las ideas de la soberania emanada del pueblo y no del derecho divino de los reyes $y$ de la legitima resistencia al tirano para llenar el vacio de poder que habia dejado la vacancia del trono por la prisión de los reyes, evolucionó hacia la idea de independencia absoluta de la metrópoli (De la Vega, 1998, p. 28). Sin embargo, el proceso de independencia no vería su final sino hasta el mes de junio de 1821 , cuando el último reducto español se rindió ante las tropas comandadas por el General Mariano Montilla.

El 11 de noviembre de 1811, como sostiene Jaime Rodriguez, Una gran multitud se congregó frente al palacio del Cabildo, donde se reunia la
Junta de Gobierno. El 'pueblo', por medio de sus representantes, los abogados Ignacio Muñoz y Nicolás Mauricio de Omaña, exigió la declaración de independencia y la abolición de la Inquisición (...) La junta de Cartagena de Indias estuvo de acuerdo (Rodríguez, 1996, p. 188). Este dia, a diferencia del 22 de mayo de 1810 cuando se creó la primera Junta de Gobierno, el pueblo cartagenero renunció al reconocimiento del Consejo de Regencia que aún atormentaba a los más radicales independentistas ${ }^{4}$.

Para los negros esclavos, libres, mulatos e indios, liberarse de esta amenaza significó una inclusión distinta en la ciudad; si bien no podian catalogarse como actores politicos, sí fueron actores sociales determinantes durante todo el proceso de levantamiento, conformación y deliberación de las Juntas de Gobierno. En otras palabras, los diversos estamentos que conformaban el pueblo cartagenero percibieron por los resultados de esta movilización, que sectores como el arrabal del barrio Getsemaní tenían capacidad de presión, al punto que en el Acta de Independencia fueron reconocidos como parte de la soberania de su pueblo.

Para Sidney Tarrow (1998) "Los descontentos encuentran oportunidades favorables para reclamar sus demandas cuando se abre el acceso institucional, cuando emergen conflictos entre las élites, cuando pueden conseguir alianzas y disminuye la capacidad represora del Estado" (p. 110). Estas dimensiones las encontramos en los hechos del 11 de noviembre, donde puede identificarse un alianza entre la élite criolla de la ciudad en cabeza de García de Toledo, el cual pidió a Juan José Solano y a Pedro Romero que se acercaran a la multitud del barrio Getsemaní y propiciaran un golpe el 14 de junio de 1810 en contra del gobernador Montes por considerarlo un "afrancesado".

Con la expulsión del gobernador Montes, se creó el batallón Lanceros de Getsemaní, integrado en su mayoría por artesanos mulatos y negros del barrio más grande de la ciudad, y el cual fue protagonista de esta movilización: "se Este día fue el preludio a la destrucción de todos los elementos de tortura que sometían a un pueblo oprimido y la abolición de la monstruosa institución de la Inquisición. Las penas generalmente impuestas por la Inquisición, consistían en multas, confiscación, destierro, flagelación, remo en galera, vergüenza pública, prisión y muerte en la hoguera. $Y$ no sólo se castigaba a los vivos sino también a los muertos, en efigie. 
trataba de un ejército que tenía toda la confianza y bendición de la élite criolla abanderada por García de Toledo" (Múnera, 1998, p. 179). Sin embargo, el desarrollo del conflicto entre los realistas y los independentistas generó una desconfianza entre los negros mulatos por las posiciones moderadas de los criollos frente a una opinión generalizada de querer romper radicalmente los lazos con la Monarquía.

La petición era unánime y bien sabida por la Junta de Gobierno de la ciudad: la única manera de mantener los lazos con la Corona, era conseguir un trato de igualdad de representación con la Junta Suprema General y Gubernativa del Reino que se estableció en territorio español. Pero el 19 de junio de 1811 , las cortes españolas reunidas en Cádiz negaron cualquier posibilidad de igualdad, afirmando que los criollos nunca alcanzarian el derecho a una representación igual a la que gozaban las provincias españolas.

Lo anterior, sumado a la decisión de negar el derecho de ciudadanía a los negros, mulatos y zambos en una decisión de 108 votos contra 36 (King en Múnera, 1998), llevaría a que Pedro Romero, ayudado de Gabriel Piñeres de Gutiérrez, abanderara la separación radical de España. Las consecuencias de este hecho marcaron una ruptura trascendental, sobre todo porque la representación de los sectores populares llevó a que estas opiniones fueran escuchadas.

La primera República, que duró hasta 1815, no vio la renuncia definitiva de la élite criolla en el poder; de hecho los abogados García de Toledo y Ayos heredaron el poderío de la élite criolla tras la muerte, en 1812, de otros dirigentes como Antonio De Narváez y José Ignacio Pombo y el lento enfriamiento de la movilización popular

$5 \quad$ Antonio Narvaéz de la Torre, según Múnera, era no sólo el hombre más respetado por las élites criollas, sino que a su inmenso prestigio entre las gentes del pueblo unía el hecho de ser tío de los hermanos De Piñeres, dirigentes del partido popular en 1812. Pombo era miembro de la Asamblea Constituyente en la cual intentaría persuadir a los criollos a aceptar la prohibición del comercio de esclavos y según el historiador Jorge Orlando Melo: "Fue probablemente el neogranadino que mejor conoció la literatura económica de su época. Ya en 1800 citaba a Adam Smith, y es evidente su familiaridad con los ilustrados y arbitristas españoles como Ward, Campillo, Jovellanos, Campomanes y Floridablanca. Entre los franceses había leído a Necker y D'Alambert, y cita con frecuencia a los norteamericanos Jefferson y Albert Gallatin". Relación de méritos y de los diversos expedientes de los legajos 925, 957 y 960 del Fondo Audiencia de Santafé, del Archivo General de Indias. que desde la teoría de la movilización de recursos de los movimientos sociales, se convirtió en una estructura de reserva ${ }^{6}$.

Por lo tanto, la lucha por alcanzar la independencia definitiva de España se encuentra en el rincón de barrios como el viejo Getsemaní o el invaluable San Sebastián; estos lugares conforman una memoria y una narrativa histórica construida a partir de acciones colectivas como la movilización popular que terminó en la firma del Acta de Independencia. En este orden de ideas, cada uno de estos sitios constituye un espacio público donde empezó a conformarse la opinión pública de acuerdo a la concepción habermasiana, la cual entiende que este "es un ámbito de nuestra vida social, en el que se puede construir algo (...) Los ciudadanos se convierten como público, cuando se reúnen y conciertan libremente, sin presiones y con la garantía de poder manifestar y publicar libremente su opinión, sobre las oportunidades de actuar según intereses generales" (Habermas, 1981, p. 53).

Tal vez el concepto de Habermas de espacio público no tuvo para el caso de estos territorios las mismas variaciones que en Europa, sin embargo, el proceso de la ilustración y la fuerte presencia en la ciudad de unas élites con cierta capacidad económica en incidencia en el subsistema administartivo, hace que ese proceso tenga ragos similares entre 1780 y 1811. Sin embargo, los elementos antes analizados nos permiten decir que se puede reconocer en la ciudad de Cartagena, un espacio no sólo de la vida social, sino uno donde el público se reunía para manifestar su opinión.

En ese sentido, las aglomeraciones en plazas y calles eran manifestaciones sujetas a presiones y a una posible censura por parte de la élite política de la época; sin embargo, el hecho mismo de la existencia de este tipo de acciones

$6 \quad$ Para Sidney Tarrow "Una vez conseguidas las reformas, o si disminuía la movilización, los militantes desaparecían en estructuras de reservas como iglesias o logias", el movimiento de acción colectiva en Cartagena logró un primer objetivo que fue forzar la declaración de independencia absoluta en 1811 , abriendo la puerta a un gran cambio estructural que se manifestó en un concepto de representación que incluía a sectores históricamente excluidos. Estos sectores pudieron expresar sus opiniones en altos cargos políticos, pero el movimiento no pudo ir más allá de esos cambios y no pudo consolidar el proceso de cambio, por el contrario entró en una nueva etapa de enfriamiento que junto a otros elementos de carácter político permitiría la reconquista en 1815. 
colectivas puede explicarse como un principio de interpretación y desarrollo del espacio público como lo define el autor antes señalado. Por lo tanto, estos espacios se fueron haciendo públicos porque reunieron en ellos un marco de consenso a través de un sentimiento emancipador de los diversos estamentos económicos y raciales que la habitaban. Lo cierto es que la Plaza de la Proclamación, lugar donde se congregó el pueblo para apoyar el Acta de Independencia de Cartagena, así como la Plaza del Pozo que fue testigo del lugar de partida de los lanceros de Getsemani que presionaron para que se firmara la Independencia absoluta, o la calle del Tablón donde se aglutinó el pueblo a leer los pasquines con mensajes libertarios, fueron espacios que sirvieron de escenario para la expresión de las primeras manifestaciones de opinión pública en la ciudad.

\section{Conclusiones}

En primer lugar, gracias a la publicidad representativa, es decir a los mensajes libertarios, la consolidación de la imprenta y el auge de los papeles públicos que activaron la movilización, la ciudad estuvo expuesta a la información. Sin adelantarnos a pensar que existía libertad de prensa, si podemos evidenciar una mayor facilidad de acceso y circulación a información diferente a la que exclusivamente se conocía a través de las gacetas oficiales que moldeaban la información a su antojo. Como menciona Renán Silva: "ya no se dirá de los papeles públicos, que simplemente son un medio de ilustración y educación, sino la forma por excelencia de fijar la opinión" (Silva, 1998, p. 106).

En segundo lugar, el papel de las élites politicas y militares fue fundamental durante ese proceso ya que, a través de la información y la publicación de la acción gubernamental, permitieron al pueblo de Cartagena formarse una percepción del destino político, económico y social de la ciudad. Sin embargo, fue necesaria una ruptura institucional que garantizara los derechos de representación como los pilares sobre los que se enmarcó la construcción de esta opinión pública política. Esa ruptura fue consecuencia directa de una movilización popular. Es decir que una acción colectiva de un puñado de mulatos y artesanos liderados por Pedro Romero, pero inspirados por algunos lideres criollos, abanderaron el 11 de noviembre del mismo año un repertorio de acción en la plaza de armas, para obligar a la Junta de Gobierno a declarar la independencia absoluta de España.

En tercer lugar, el camino hacia la modernidad política sólo fue posible tras la ruptura con la Monarquía española; es decir, después de la consolidación de esa Primera República que duró hasta 1815, pero que mostró avances significativos frente a lo que vivia la ciudad bajo el Antiguo Régimen. Existe certeza de que mulatos y artesanos ocuparon altos cargos públicos a partir del año 1812. Incluso Pedro Romero, líder artesano de los lanceros de Getsemaní, fue elegido en la convención que elaboró la constitución del Estado, un hecho sin precedentes que incluía a sectores históricamente excluidos de cualquier asunto politico.

Finalmente, la ciudad de Cartagena de Indias se fue construyendo como una esfera de opinión pública política donde la sociedad pudo circular, debatir y opinar sobre decisiones gubernamentales que los afectaban directamente en calles, plazas, barrios y demás espacios públicos de la ciudad, que a través del mecanismo de la movilización logró expresar, incidir e incluir las ideas y opiniones del pueblo en la herramienta última de este proceso como fue el Acta de Independencia del 11 de noviembre de 1811 . 


\section{Referencias}

1. Boladeras, C. M. (2001). La opinión pública en Habermas. Barcelona:

Universidad de Barcelona.

2. Del Real Torres, A. (1946). Biografía de Cartagena 1533-1945. Cartagena: Imp departamental.

3. De La Vega Sourdis, A. (1998) Cartagena de Indias durante la Primera República 1810-1815. Bogotá: Banco de La República Ediciones.

4. Guerra, F. X. \& Lempiere, A. (1998). Los espacios públicos en Iberoamérica Ambigüedades y problemas, Siglos XVIII.XIX. México D.F: Fondo de Cultura Económica.

5. Guerra, J. F. \& Lemperiere, A. (1998). "La Publicidad de La Junta Central Española (1808-1810)". Los espacios públicos en Iberoamérica, ambigüedades y problemas, Siglos XVIII, XIX. México D.F: Fondo de Cultura Económica.

6. Guerra, J. F. \& Lemperiere, A. (1998). "Prácticas de lectura, ámbitos privados y formación de un espacio público moderno. Nueva Granada a finales del Antiguo Régimen". Los espacios públicos en Iberoamérica, ambigüedades y problemas, Siglos XVIII, XIX. México D.F: Fondo de Cultura Económica.

7. Habermas, J. (1981). Teoría de la acción comunicativa. Madrid: Edición Taurus.

8. Labatut, P. (1812). El efímero de Cartagena",Proclama del Coronel Pedro Labatut. 1812.

9. Lemaitre, E. (1983). Historia general de Cartagena. Bogotá: Banco de la República.

10. Morales Ballestas, R. (2003).

Cartagena de Indias relatos de la vida cotidiana y otras historias. Colombia: Universidad Libre Ediciones.
11. Múnera, A. (1998). El Fracaso de la Nación, región, clase y raza en el Caribe colombiano (1717-1810). Bogotá: El Ancora Editores.

12. Muñoz, A. A. (1992). Opinión pública y comunicación política. Madrid: Eudema Ediciones.

13. Ortiz, J., Quiroz, P. \& Roman, R. (2001). Desorden en la Plaza, modernización y memoria urbana en Cartagena. Medellín: Editorial Lealon.

14. Ripoll, M. T. (2006). La élite en Cartagena y su tránsito a la República, revolución política sin renovación social. Bogotá: Ediciones Uniandes.

15. Rivas Funes, J. M. (1995). El asociacionismo y la redefinición de los espacios políticos. España: Revista de Estudios Políticos.

16. Roca Meisel, A. (2003). ¿Situado o Contrabando?: la base económica de Cartagena de Indias a fines del siglo de las luces. Cartagena: Cuadernos de historia económica y empresarial, Banco de la República.

17. Rodríguez, J. (1996). La

Independencia de la América Española. México D.F: Fondo de Cultura Económica.

18. Tarrow, S. (2004). El poder en movimiento, los movimientos sociales, la acción colectiva y la política. Madrid: Editorial Alianza. 


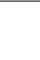

